\title{
TWO NEW REVERSES OF YOUNG'S INEQUALITY
}

\author{
SEVER S. DRAGOMIR ${ }^{1,2}$
}

\begin{abstract}
In this paper we obtain two new reverses of Young's inequality.
\end{abstract}

\section{INTRODUCTION}

The famous Young inequality for scalars says that if $a, b>0$ and $\nu \in$ $[0,1]$, then

$$
a^{1-\nu} b^{\nu} \leq(1-\nu) a+\nu b
$$

with equality if and only if $a=b$. The inequality (1.1) is also called $\nu$ weighted arithmetic-geometric mean inequality.

We recall that Specht's ratio is defined by [9]

$$
S(h):=\left\{\begin{array}{l}
\frac{h^{\frac{1}{h-1}}}{e \ln \left(h^{\frac{1}{h-1}}\right)} \text { if } h \in(0,1) \cup(1, \infty), \\
1 \text { if } h=1 .
\end{array}\right.
$$

It is well known that $\lim _{h \rightarrow 1} S(h)=1, S(h)=S\left(\frac{1}{h}\right)>1$ for $h>0, h \neq 1$. The function is decreasing on $(0,1)$ and increasing on $(1, \infty)$.

The following inequality provides a refinement and a multiplicative reverse for Young's inequality

$$
S\left(\left(\frac{a}{b}\right)^{r}\right) a^{1-\nu} b^{\nu} \leq(1-\nu) a+\nu b \leq S\left(\frac{a}{b}\right) a^{1-\nu} b^{\nu},
$$

where $a, b>0, \nu \in[0,1], r=\min \{1-\nu, \nu\}$.

The second inequality in (1.3) is due to Tominaga [10] while the first one is due to Furuichi [3].

In [10], Tominaga also proved the following additive reverse of Young's inequality

$$
(1-\nu) a+\nu b-a^{1-\nu} b^{\nu} \leq S\left(\frac{a}{b}\right) L(a, b)
$$

2000 Mathematics Subject Classification. 26D15; 26D10.

Key words and phrases. Young's Inequality, Convex functions, Arithmetic meanGeometric mean inequality. 
where $a, b>0, \nu \in[0,1]$ and $L(a, b)$ is the logarithmic mean, namely

$$
L(a, b):=\left\{\begin{array}{cll}
\frac{b-a}{\ln b-\ln a} & \text { if } & a \neq b \\
b & \text { if } & a=b .
\end{array}\right.
$$

Kittaneh and Manasrah [6], [7] provided a refinement and an additive reverse for Young inequality as follows:

$$
r(\sqrt{a}-\sqrt{b})^{2} \leq(1-\nu) a+\nu b-a^{1-\nu} b^{\nu} \leq R(\sqrt{a}-\sqrt{b})^{2}
$$

where $a, b>0, \nu \in[0,1], r=\min \{1-\nu, \nu\}$ and $R=\max \{1-\nu, \nu\}$.

We also consider the Kantorovich's ratio defined by

$$
K(h):=\frac{(h+1)^{2}}{4 h}, h>0 .
$$

The function $K$ is decreasing on $(0,1)$ and increasing on $[1, \infty), K(h) \geq 1$ for any $h>0$ and $K(h)=K\left(\frac{1}{h}\right)$ for any $h>0$.

The following multiplicative refinement and reverse of Young inequality in terms of Kantorovich's ratio holds

$$
K^{r}\left(\frac{a}{b}\right) a^{1-\nu} b^{\nu} \leq(1-\nu) a+\nu b \leq K^{R}\left(\frac{a}{b}\right) a^{1-\nu} b^{\nu}
$$

where $a, b>0, \nu \in[0,1], r=\min \{1-\nu, \nu\}$ and $R=\max \{1-\nu, \nu\}$.

The first inequality in (1.7) was obtained by Zou et al. in [11] while the second by Liao et al. [8].

In [11] the authors also showed that $K^{r}(h) \geq S\left(h^{r}\right)$ for $h>0$ and $r \in\left[0, \frac{1}{2}\right]$ implying that the lower bound in (1.7) is better than the lower bound from (1.3).

In the recent paper [1] we obtained the following reverses of Young's inequality as well:

$$
0 \leq(1-\nu) a+\nu b-a^{1-\nu} b^{\nu} \leq \nu(1-\nu)(a-b)(\ln a-\ln b)
$$

and

$$
1 \leq \frac{(1-\nu) a+\nu b}{a^{1-\nu} b^{\nu}} \leq \exp \left[4 \nu(1-\nu)\left(K\left(\frac{a}{b}\right)-1\right)\right],
$$

where $a, b>0, \nu \in[0,1]$.

It has been shown in [1] that there is no ordering for the upper bounds of the quantity $(1-\nu) a+\nu b-a^{1-\nu} b^{\nu}$ as provided by the inequalities (1.5) and (1.8). The same conclusion is true for the upper bounds of the quantity $\frac{(1-\nu) a+\nu b}{a^{1-\nu} b^{\nu}}$ incorporated in the inequalities (1.3), (1.7) and (1.9).

In [2] we also proved the following refinements and reverses of Young's inequality:

$$
\frac{1}{2} \nu(1-\nu)(\ln a-\ln b)^{2} \min \{a, b\} \leq(1-\nu) a+\nu b-a^{1-\nu} b^{\nu}
$$




$$
\leq \frac{1}{2} \nu(1-\nu)(\ln a-\ln b)^{2} \max \{a, b\}
$$

and

$$
\begin{array}{r}
\exp \left[\frac{1}{2} \nu(1-\nu)\left(1-\frac{\min \{a, b\}}{\max \{a, b\}}\right)^{2}\right] \leq \frac{(1-\nu) a+\nu b}{a^{1-\nu} b^{\nu}} \\
\leq \exp \left[\frac{1}{2} \nu(1-\nu)\left(\frac{\max \{a, b\}}{\min \{a, b\}}-1\right)^{2}\right]
\end{array}
$$

for any $a, b>0$ and $\nu \in[0,1]$.

In [2] we also showed that there is no ordering between the upper bounds of the quantity $(1-\nu) a+\nu b-a^{1-\nu} b^{\nu}$ provided by (1.8) and (1.10) respectively. The same conclusion applies for the upper bounds of the quantity $\frac{(1-\nu) a+\nu b}{a^{1-\nu} b^{\nu}}$ provided by (1.9) and (1.11).

Now, if $a, b \in[m, M]$ with $0<m<M$ and we want upper bounds for the quotient $\frac{(1-\nu) a+\nu b}{a^{1-\nu} b^{\nu}}$ in terms of $m, M$ the we can use either of the inequalities (1.3), (1.7), (1.9) or (1.11).

Indeed, since, for instance, by using the properties of Specht's ratio we have

$$
\max _{a, b \in[m, M]} S\left(\frac{a}{b}\right)=\max \left\{S\left(\frac{m}{M}\right), S\left(\frac{M}{m}\right)\right\}=S\left(\frac{M}{m}\right),
$$

then by (1.3) we have

$$
\frac{(1-\nu) a+\nu b}{a^{1-\nu} b^{\nu}} \leq S\left(\frac{M}{m}\right)
$$

for any $a, b \in[m, M]$.

By (1.7) we get

$$
\frac{(1-\nu) a+\nu b}{a^{1-\nu} b^{\nu}} \leq K^{R}\left(\frac{M}{m}\right)
$$

for any $a, b \in[m, M]$ and similar results from (1.9) and (1.11).

In the additive case, we have by (1.4) that

$$
(1-\nu) a+\nu b-a^{1-\nu} b^{\nu} \leq S\left(\frac{M}{m}\right) L(M, m)
$$

for any $a, b \in[m, M]$ while by (1.5)

$$
(1-\nu) a+\nu b-a^{1-\nu} b^{\nu} \leq R(\sqrt{M}-\sqrt{m})^{2}
$$

for any $a, b \in[m, M]$ and similar results from (1.8) and (1.10).

Motivated by the above results we establish in this paper two new reverses of Young's inequality (1.1) assuming that the positive numbers $a$, $b$ are bounded below and above by $m$ and $M$, respectively. The obtained results are shown to improve the inequalities (1.12)-(1.15) and the other multiplicative or additive reverses mentioned above. 


\section{Inequalities for Convex Functions}

In this section we establish some reverse inequalities in the general setting of convex functions. These results will then be used to obtain our additive and multiplicative reverses of Young's inequality (1.1).

Consider a convex function $f: I \subset \mathbb{R} \rightarrow \mathbb{R}$ defined on the interval $I$ of the real line $\mathbb{R}$ and two distinct numbers $a, b \in I$ with $a<b$. We denote by $[a, b]$ the closed interval defined by $\{(1-t) a+t b, t \in[0,1]\}$. We also define the functional of interval

$$
\Delta_{f}([a, b] ; t):=(1-t) f(a)+t f(b)-f((1-t) a+t b) \geq 0
$$

where $a, b \in I$ with $a<b$ and $t \in[0,1]$.

We have the following key lemma that is of interest in itself:

Lemma 1. Let $f: I \subset \mathbb{R} \rightarrow \mathbb{R}$ be a convex function on the interval $I$. Then for each $a, b \in I$ with $a<b$ and $c \in[a, b]$ we have

$$
(0 \leq) \Delta_{f}([a, c] ; t)+\Delta_{f}([c, b] ; t) \leq \Delta_{f}([a, b] ; t)
$$

for each $t \in[0,1]$, i.e., the functional $\Delta_{f}(\cdot ; t)$ is superadditive as a function of interval.

If $[c, d] \subset[a, b]$, then

$$
(0 \leq) \Delta_{f}([c, d] ; t) \leq \Delta_{f}([a, b] ; t)
$$

for each $t \in[0,1]$, i.e., the functional $\Delta_{f}(\cdot ; t)$ is nondecreasing as a function of interval.

Proof. Let $c=(1-s) a+s b$ with $s \in(0,1)$. For $t \in(0,1)$ we have

$\Delta_{f}([c, b] ; t)=(1-t) f((1-s) a+s b)+t f(b)-f((1-t)[(1-s) a+s b]+t b)$

and

$\Delta_{f}([a, c] ; t)=(1-t) f(a)+t f((1-s) a+s b)-f((1-t) a+t[(1-s) a+s b])$

giving that

$$
\begin{aligned}
& \Delta_{f}([a, c] ; t)+\Delta_{f}([c, b] ; t)-\Delta_{f}([a, b] ; t) \\
& =f((1-s) a+s b)+f((1-t) a+t b) \\
& -f((1-t)(1-s) a+[(1-t) s+t] b)-f((1-t s) a+t s b) .
\end{aligned}
$$

Since $\varphi$ is convex on $I$ then for any $a \in I$ the function $\psi: I \backslash\{a\} \rightarrow \mathbb{R}$

$$
\psi(t):=\frac{\varphi(t)-\varphi(a)}{t-a}
$$

is monotonic nondecreasing where is defined. Utilising this property repeatedly we have

$$
\frac{\varphi\left(t_{1}\right)-\varphi\left(t_{2}\right)}{t_{1}-t_{2}} \leq \frac{\varphi\left(s_{1}\right)-\varphi\left(t_{2}\right)}{s_{1}-t_{2}}=\frac{\varphi\left(t_{2}\right)-\varphi\left(s_{1}\right)}{t_{2}-s_{1}}
$$




$$
\leq \frac{\varphi\left(s_{2}\right)-\varphi\left(s_{1}\right)}{s_{2}-s_{1}}=\frac{\varphi\left(s_{1}\right)-\varphi\left(s_{2}\right)}{s_{1}-s_{2}} .
$$

Therefore, for a convex function $\varphi: I \subset \mathbb{R} \rightarrow \mathbb{R}$, where $I$ is an interval, and any real numbers $t_{1}, t_{2}, s_{1}$ and $s_{2}$ from $I$ and with the properties that $t_{1} \leq s_{1}$ and $t_{2} \leq s_{2}$ we have that

$$
\frac{\varphi\left(t_{1}\right)-\varphi\left(t_{2}\right)}{t_{1}-t_{2}} \leq \frac{\varphi\left(s_{1}\right)-\varphi\left(s_{2}\right)}{s_{1}-s_{2}}
$$

Consider the function $\varphi:[0,1] \rightarrow \mathbb{R}$ given by $\varphi(t):=f((1-t) a+t b)$. Since $f$ is convex on $I$ it follows that $\varphi$ is convex on $[0,1]$. Now, if we consider for given $t, s \in(0,1)$

$$
t_{1}:=t s<s=: s_{1} \text { and } t_{2}:=t<t+(1-t) s=: s_{2},
$$

then we have

$$
\varphi\left(t_{1}\right)=f((1-t s) a+t s b), \varphi\left(t_{2}\right)=f((1-t) a+t b)
$$

giving that

$$
\frac{\varphi\left(t_{1}\right)-\varphi\left(t_{2}\right)}{t_{1}-t_{2}}=\frac{f((1-t s) a+t s b)-f((1-t) a+t b)}{t(s-1)} .
$$

Also

$$
\varphi\left(s_{1}\right)=f((1-s) a+s b), \varphi\left(s_{2}\right)=f((1-t)(1-s) a+[(1-t) s+t] b)
$$

giving that

$\frac{\varphi\left(s_{1}\right)-\varphi\left(s_{2}\right)}{s_{1}-s_{2}}=\frac{f((1-s) a+s b)-f((1-t)(1-s) a+[(1-t) s+t] b)}{t(s-1)}$.

Utilising the inequality (2.5) and multiplying with $t(s-1)<0$ we deduce the inequality

$$
\begin{aligned}
& f((1-t s) a+t s b)-f((1-t) a+t b) \\
& \geq f((1-s) a+s b)-f((1-t)(1-s) a+[(1-t) s+t] b) .
\end{aligned}
$$

Finally, by (2.4) and (2.6) we get the desired result (2.2).

Applying repeatedly the superadditivity property we have for $[c, d] \subset$ $[a, b]$ that

$$
\Delta_{f}([a, c] ; t)+\Delta_{f}([c, d] ; t)+\Delta_{f}([d, b] ; t) \leq \Delta_{f}([a, b] ; t)
$$

giving that

$$
0 \leq \Delta_{f}([a, c] ; t)+\Delta_{f}([d, b] ; t) \leq \Delta_{f}([a, b] ; t)-\Delta_{f}([c, d] ; t),
$$

which proves (2.3).

For any $a, b \in I$ we define

$$
\Delta_{f}(a, b ; t):=(1-t) f(a)+t f(b)-f((1-t) a+t b)
$$

for $t \in[0,1]$. 
Theorem 1. Let $f:[m, M] \rightarrow \mathbb{R}$ be a convex functions on the closed interval $[m, M]$. Then for any $a, b \in[m, M]$ we have

$$
(0 \leq) \Delta_{f}(a, b ; t) \leq \max \left\{\Delta_{f}(m, M ; t), \Delta_{f}(m, M ; 1-t)\right\}
$$

for any $t \in[0,1]$.

Proof. If $a<b$, then $[a, b] \subset[m, M]$ and by (2.3) we have

$$
\Delta_{f}(a, b ; t)=\Delta_{f}([a, b] ; t) \leq \Delta_{f}(m, M ; t)
$$

for any $t \in[0,1]$.

If $b<a$, then $[b, a] \subset[m, M]$ and by (2.3) we have

$$
\begin{aligned}
\Delta_{f}(a, b ; t) & =(1-t) f(a)+t f(b)-f((1-t) a+t b) \\
& =t f(b)+(1-t) f(a)-f(t b+(1-t) a) \\
& =[1-(1-t)] f(b)+(1-t) f(a)-f([1-(1-t)] b+(1-t) a) \\
& =\Delta_{f}([b, a] ; 1-t) \leq \Delta_{f}(m, M ; 1-t)
\end{aligned}
$$

and the inequality (2.8) is proved.

Corollary 1. Let $f:[m, M] \rightarrow \mathbb{R}$ be a convex functions on the closed interval $[m, M]$. Then for any $a, b \in[m, M]$ we have

$$
(0 \leq) \frac{f(a)+f(b)}{2}-f\left(\frac{a+b}{2}\right) \leq \frac{f(m)+f(M)}{2}-f\left(\frac{m+M}{2}\right) .
$$

The proof follows from (2.8) by taking $t=\frac{1}{2}$.

Corollary 2. Let $f:[m, M] \rightarrow \mathbb{R}$ be a convex functions on the closed interval $[m, M]$. Then for any $a, b \in[m, M]$ we have

$$
\begin{aligned}
& (0 \leq) \frac{f(a)+f(b)}{2}-\frac{1}{b-a} \int_{a}^{b} f(x) d x \\
& \leq \frac{f(m)+f(M)}{2}-\frac{1}{M-m} \int_{m}^{M} f(x) d x .
\end{aligned}
$$

Proof. If $a<b$, then $[a, b] \subset[m, M]$ and by (2.3) we have

$$
\Delta_{f}(a, b ; t) \leq \Delta_{f}(m, M ; t)
$$

for any $t \in[0,1]$.

Integrating this inequality over $t$ on $[0,1]$ we get

$$
\int_{0}^{1} \Delta_{f}(a, b ; t) d t \leq \int_{0}^{1} \Delta_{f}(m, M ; t) d t .
$$

and since

$$
\int_{0}^{1} \Delta_{f}(a, b ; t) d t=\frac{f(a)+f(b)}{2}-\frac{1}{b-a} \int_{a}^{b} f(x) d x
$$


and

$$
\int_{0}^{1} \Delta_{f}(m, M ; t) d t=\frac{f(m)+f(M)}{2}-\frac{1}{M-m} \int_{m}^{M} f(x) d x,
$$

then we get the desired result (2.10).

If $b<a$, then

$$
\Delta_{f}(a, b ; t) \leq \Delta_{f}(m, M ; 1-t)
$$

for any $t \in[0,1]$.

Integrating this inequality over $t$ on $[0,1]$ and since

$$
\int_{0}^{1} \Delta_{f}(m, M ; 1-t) d t=\frac{f(m)+f(M)}{2}-\frac{1}{M-m} \int_{m}^{M} f(x) d x
$$

we also obtain the desired result (2.10).

Corollary 3. Let $f:[m, M] \rightarrow \mathbb{R}$ be a convex functions on the closed interval $[m, M]$. Then for any $a, b \in[m, M]$ and $t \in[0,1]$ we have

$$
\begin{aligned}
& (0 \leq) \frac{f((1-t) a+t b)+f((1-t) b+t a)}{2}-f\left(\frac{a+b}{2}\right) \\
& \leq \frac{f(m)+f(M)}{2}-f\left(\frac{m+M}{2}\right) .
\end{aligned}
$$

The proof follows by $(2.9)$ on replacing $a$ with $(1-t) a+t b$ and $b$ with $(1-t) b+t a$.

\section{Reverses of Young's InEquality}

We have the following reverse additive version of Young's inequality:

Theorem 2. If $a, b \in[m, M] \subset(0, \infty)$ and $\nu \in[0,1]$, then we have

$$
(0 \leq)(1-\nu) a+\nu b-a^{1-\nu} b^{\nu} \leq \max \left\{\iota_{m, M}(\nu), \iota_{m, M}(1-\nu)\right\}
$$

where

$$
\iota_{m, M}(\nu):=(1-\nu) m+\nu M-m^{1-\nu} M^{\nu} .
$$

Proof. Let $[m, M] \subset(0, \infty)$ and consider the interval $[\ln m, \ln M]$. Consider the convex function $f:[\ln m, \ln M] \rightarrow(0, \infty), f(x)=\exp x$. Then by $(2.8)$ we have for any $x, y \in[\ln m, \ln M]$ and $\nu \in[0,1]$ that

$$
\begin{aligned}
& (1-\nu) \exp (x)+\nu \exp (y)-\exp ((1-\nu) x+\nu y) \\
& \leq \max \{(1-\nu) \exp (\ln m)+\nu \exp (\ln M)-\exp ((1-\nu) \ln m+\nu \ln M), \\
& \nu \exp (\ln m)+(1-\nu) \exp (\ln M)-\exp (\nu \ln m+(1-\nu) \ln M)\} \\
& =\max \left\{(1-\nu) m+\nu M-m^{1-\nu} M^{\nu}, \nu m+(1-\nu) M-m^{\nu} M^{1-\nu}\right\} .
\end{aligned}
$$

If $a, b \in[m, M]$ then by taking $x=\ln a, y=\ln b \in[\ln m, \ln M]$ in (3.3) we get the inequality in (3.1). 
Remark 1. By Tominaga's inequality (1.4) we have

$$
(1-\nu) m+\nu M-m^{1-\nu} M^{\nu} \leq S\left(\frac{m}{M}\right) L(m, M)
$$

and

$$
(1-\nu) M+\nu m-M^{1-\nu} m^{\nu} \leq S\left(\frac{M}{m}\right) L(M, m)
$$

giving that

$$
\max \left\{\iota_{m, M}(\nu), \iota_{m, M}(1-\nu)\right\} \leq S\left(\frac{M}{m}\right) L(M, m) .
$$

Therefore by (3.1) and (3.4) we have

$$
\begin{aligned}
(1-\nu) a+\nu b-a^{1-\nu} b^{\nu} & \leq \max \left\{\iota_{m, M}(\nu), \iota_{m, M}(1-\nu)\right\} \\
& \leq S\left(\frac{M}{m}\right) L(M, m)
\end{aligned}
$$

for any $a, b \in[m, M]$ and $\nu \in[0,1]$.

This is a refinement of (1.14) from the introduction.

If we use (3.1) and (1.5, (1.8) and (1.10), then we get

$$
\begin{gathered}
(1-\nu) a+\nu b-a^{1-\nu} b^{\nu} \leq \max \left\{\iota_{m}, M(\nu), \iota_{m, M}(1-\nu)\right\} \leq R(\sqrt{M}-\sqrt{m})^{2}, \\
\begin{aligned}
(1-\nu) a+\nu b-a^{1-\nu} b^{\nu} & \leq \max \left\{\iota_{m, M}(\nu), \iota_{m, M}(1-\nu)\right\} \\
& \leq \nu(1-\nu)(M-m)(\ln M-\ln m)
\end{aligned}
\end{gathered}
$$

and

$$
\begin{aligned}
(1-\nu) a+\nu b-a^{1-\nu} b^{\nu} & \leq \max \left\{\iota_{m, M}(\nu), \iota_{m, M}(1-\nu)\right\} \\
& \leq \frac{1}{2} \nu(1-\nu)(\ln M-\ln m)^{2} M
\end{aligned}
$$

for any $a, b \in[m, M]$ and $\nu \in[0,1]$, respectively.

We also have the following multiplicative reverse of Young's inequality:

Theorem 3. If $a, b \in[m, M] \subset(0, \infty)$ and $\nu \in[0,1]$, then we have

$$
(1 \leq) \frac{(1-\nu) a+\nu b}{a^{1-\nu} b^{\nu}} \leq \max \left\{\kappa_{m, M}(\nu), \kappa_{m, M}(1-\nu)\right\}
$$

where

$$
\kappa_{m, M}(\nu):=\frac{(1-\nu) m+\nu M}{m^{1-\nu} M^{\nu}} .
$$

Proof. Consider the convex function $f:[m, M] \subset(0, \infty) \rightarrow \mathbb{R}, f(x)=$ $-\ln x$. Then by $(2.8)$ we have

$$
\begin{aligned}
& (0 \leq) \ln ((1-\nu) a+\nu b)-(1-\nu) \ln a-\nu \ln b \\
& \leq \max \{\ln ((1-\nu) m+\nu M)-(1-\nu) \ln m-\nu \ln M, \\
& \ln ((1-\nu) M+\nu m)-(1-\nu) \ln M-\nu \ln m\}
\end{aligned}
$$


for any $a, b \in[m, M]$ and $\nu \in[0,1]$.

This inequality is equivalent to

$(0 \leq) \ln \left(\frac{(1-\nu) a+\nu b}{a^{1-\nu} b^{\nu}}\right) \leq \max \left\{\ln \left(\frac{(1-\nu) m+\nu M}{m^{1-\nu} M^{\nu}}\right), \ln \left(\frac{(1-\nu) M+\nu m}{M^{1-\nu} m^{\nu}}\right)\right\}$,

which proves the inequality in (3.9).

Corollary 4. For any $a, b \in[m, M] \subset(0, \infty)$ we have

$$
(1 \leq) \frac{a+b}{2 \sqrt{a b}} \leq \frac{m+M}{2 \sqrt{m M}}
$$

Remark 2. By utilising (3.9) and the multiplicative reverses of Young's inequality incorporated in (1.3), (1.7), (1.9) and (1.11), we get

$$
\begin{aligned}
\frac{(1-\nu) a+\nu b}{a^{1-\nu} b^{\nu}} \leq \max \left\{\kappa_{m, M}(\nu), \kappa_{m, M}(1-\nu)\right\} \leq S\left(\frac{M}{m}\right), \\
\frac{(1-\nu) a+\nu b}{a^{1-\nu} b^{\nu}} \leq \max \left\{\kappa_{m, M}(\nu), \kappa_{m, M}(1-\nu)\right\} \leq K^{R}\left(\frac{M}{m}\right) \\
\frac{(1-\nu) a+\nu b}{a^{1-\nu} b^{\nu}} \leq \max \left\{\kappa_{m, M}(\nu), \kappa_{m, M}(1-\nu)\right\} \\
\leq \exp \left[4 \nu(1-\nu)\left(K\left(\frac{M}{m}\right)-1\right)\right]
\end{aligned}
$$

and

$$
\begin{aligned}
\frac{(1-\nu) a+\nu b}{a^{1-\nu} b^{\nu}} & \leq \max \left\{\kappa_{m, M}(\nu), \kappa_{m, M}(1-\nu)\right\} \\
& \leq \exp \left[\frac{1}{2} \nu(1-\nu)\left(\frac{M}{m}-1\right)^{2}\right]
\end{aligned}
$$

for any $a, b \in[m, M]$ and $\nu \in[0,1]$, respectively.

Acknowledgement. The author would like to thank the anonymous referee for valuable suggestions that have been implemented in the final version of the paper.

\section{REFERENCES}

[1] S. S. Dragomir, A Note on Young's Inequality, Preprint, RGMIA Res. Rep. Coll. 18 (2015), Art. 126. [http://rgmia.org/papers/v18/v18a126.pdf] .

$[2]$ S. S. Dragomir, A note on new refinements and reverses of Young's inequality, Preprint RGMIA Res. Rep. Coll. 18 (2015), Art. 131. [http://rgmia.org/papers/v18/v18a131.pdf] .

[3] S. Furuichi, Refined Young inequalities with Specht's ratio, Journal of the Egyptian Mathematical Society 20(2012), 46-49.

[4] S. Furuichi, On refined Young inequalities and reverse inequalities, J. Math. Inequal., 5 (2011), 21-31.

[5] S. Furuichi and N. Minculete, Alternative reverse inequalities for Young's inequality, J. Math Inequal. 5 (2011), Number 4, 595-600. 
[6] F. Kittaneh and Y. Manasrah, Improved Young and Heinz inequalities for matrix, J. Math. Anal. Appl., 361 (2010), 262-269

[7] F. Kittaneh and Y. Manasrah, Reverse Young and Heinz inequalities for matrices, Linear Multilinear Algebra., 59 (2011), 1031-1037.

[8] W. Liao, J. Wu and J. Zhao, New versions of reverse Young and Heinz mean inequalities with the Kantorovich constant, Taiwanese J. Math. 19 (2015), No. 2, pp. 467-479.

[9] W. Specht, Zer Theorie der elementaren Mittel, Math. Z., 74 (1960), pp. 91-98.

[10] M. Tominaga, Specht's ratio in the Young inequality, Sci. Math. Japon., 55 (2002), 583-588.

[11] G. Zuo, G. Shi and M. Fujii, Refined Young inequality with Kantorovich constant, J. Math. Inequal., 5 (2011), 551-556.

${ }^{1}$ Mathematics, College of Engineering \& Science

Victoria University, PO BOX 14428

Melbourne City, MC 8001, Australia.

E-mail address: sever.dragomir@vu.edu.au

$U R L$ : http://rgmia.org/dragomir

${ }^{2}$ School of Computer Science \& Applied Mathematics,

UNIVERSity OF THE WitWATERSRAND,

Private Bag 3, Johannesburg 2050, South Africa 